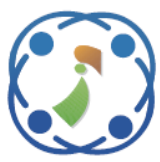

\title{
Limited Feedback Based on Hybrid Beamforming for Outdoor Environments Towards 5G Networks
}

\author{
Mohammed K. Hussein ${ }^{1 *}$ \\ Nasser N. Khamiss ${ }^{1}$ \\ ${ }^{1}$ Information and Communication Engineering, Al-Nahrain University, Baghdad, Iraq \\ * Corresponding author's Email: mohammed.khudhur@gmail.com
}

\begin{abstract}
The 5G approximately provides 1000x higher mobile data traffic and 10 to 100x numbers of connected devices than the $4 \mathrm{G}$. The $5 \mathrm{G}$ is becoming a reality after deploying it by some of the main cities in the world. Millimeterwave (mm-wave) communications play a crucial role in the $5 \mathrm{G}$ networks owing to its enormous available bandwidth. The hybrid beamforming (HBF) presents better multiplexing gains than analog beamforming. It is a trade-off between cost and performance. As a result, the HBF is a promising approach that exploits the small digital beamforming and the high analog beamforming to meet these challenges. This study proposes and simulates a limited feedback hybrid beamforming for outdoor environments, including the analog precoding/combining based on a quantization codebook and the digital precoding based on Lagrangian optimization with the mathematical model in detail compared with other algorithms in the literature. It achieves an increase in the spectral efficiency satisfied, such as at 20dB SNR, it is approximately $11.75 \mathrm{bps} / \mathrm{Hz}$ while the other solutions are $11.18,11.4$, and $11.8 \mathrm{bps} / \mathrm{Hz}$. Also, it presents work well in all possible and satisfies the quality of the results for all cases, such as increasing the number of users, number of multi-path, the SNR, and the BS antennas. Also, it offers the BER approximately $10^{-4}$ at $10.3 \mathrm{SNR}$, better than other solutions.
\end{abstract}

Keywords: Massive MIMO, Millimeter-wave, Hybrid beamforming, Lagrangian optimization.

\section{Introduction}

Communication network generations have emerged approximately every ten years due to the rapid growth of mobile and wireless networks since 1980 [1]. With each generation, some techniques and new features support a world of mobile and wireless networks. The 5th generation $(5 \mathrm{G})$ trend is a Wireless World Wide Web (WWWW) [2]. The 5G provides 1000x higher mobile data traffic and 10 to $100 \mathrm{x}$ numbers of connected devices than the 4G [3]. The $5 \mathrm{G}$ is becoming a reality after deploying it by some of the main cities in the world. Millimeter-wave (mm-wave) communications play a crucial role in the 5G networks owing to its enormous available bandwidth [4]. Recent studies have confirmed a need for a fundamental change in the system architecture and radio technology to fulfill $5 \mathrm{G}$ goals $[5,6]$. The three aspects can enhance the throughput according to the Shannon-Hartley Theorem: 1) The use of an extra spectrum leads to an increase in the data capacity. 2) The increase in the number of antennas at both sides adds spatial links between a transmitter (Tx) and receiver (Rx) [7]. 3) Cell splitting can enhance data capacity and coverage areas.

The wavelength of mm-wave bands is very small, resulting in a propagation loss compared to the microwave bands. However, the Massive MIMO makes mm-wave bands suitable by deploying more antennas in a small physical area to achieve high-gain antennas [8-12].

Beamforming adjusts a radiation pattern for the base station (BS) to add signals in desired directions and null in other directions. Therefore, the narrow and directional beams play a prominent role in eliminating interference and focusing antenna energy in the desired direction. The change of phase or amplitude requires manipulating different signals from different antennas to achieve that. Beamforming architecture can be classified into three main 
categories: analog, digital, and hybrid beamforming [13].

The analog beamforming (ABF) is an energyefficient solution based on inexpensive phase shifters, facilitating beam steering. However, it is limited to one data stream, leading to performance limitations in a multi-user scenario. Digital beamforming (DBF) adjusts both the magnitude and phase for each antenna element to provide the highest flexibility according to the beamforming concept. The ideal of the DBF achieves a full spatial multiplexing gain. In more detail, the DBF works at sub-6 GHz systems that require a dedicated RF chain equipped with a single antenna resulting in more cost and consumption of energy. It is because that the DBF is unpractical in Massive MIMO scenarios [11] and [14-15]. The hybrid beamforming (HBF) presents better multiplexing gains than analog beamforming. It is a trade-off between cost and performance. As a result, the HBF is a promising approach that exploits the small digital beamforming and the high analog beamforming to meet these challenges [16-22].

\section{Literature survey}

Existing research efforts have continued to deploy new algorithms or improve existing ones for achieving the $5 \mathrm{G}$ goals. A body of research has focused extensively on hybrid beamforming and heterogeneous networks (HetNets). Here, we examine some of the related research on these subjects. The authors in [23] presented a Kalman algorithm and compared it with a minimum mean square error algorithm (MMSE) and a zero-forcing (ZF) algorithm. The results showed that the Kalman algorithm outperformed other algorithms. However, the authors did not explain the algorithm in detail and did not indicate the method in an analog stage. In other words, this algorithm consists of two stages, in which the Kalman algorithm is only represented a digital stage. Finally, there is no mathematical model. In [24], the authors evaluated the three algorithms: the Kalman algorithm, the MMSE algorithm, and the ZF algorithm. The results showed that the Kalman algorithm is better than other algorithms. However, some mathematical equations are showing suddenly without any relation sequence already. Finally, the Kalman algorithm is used as the digital stage and a codebook as the analog stage. The authors in [25] presented the ZF algorithm as the digital stage and then compared it with full digital precoding. However, the ZF algorithm lacks the work in a multi-user environment. In [26], the author proposed the MMSE algorithm as the digital stage, compared it with the $\mathrm{ZF}$ algorithm, and presented a codebook as the analog stage. However, the authors did not indicate the method used in obtaining the codebook. The authors in [27] proposed the MMSE algorithm based on a manifold optimization for a single-user environment. This method is considered a non-linear solution with computational complexity in addition to impractical for the multi-user environment.

This study aims to integrate key enabler technologies of the $5 \mathrm{G}$ such as mm-wave, Massive MIMO, and small-cells. After that, we propose solutions for the issues and challenges of this integration. As a result, a packet of advantages is obtained, including an increased network throughput and system capacity. Simultaneously, it enhances spectral and energy efficiency. This study develops a new hybrid beamforming algorithm for outdoor environments, working in the $5 \mathrm{G}$ networks to achieve the desired goals. The contribution of this study is to design and simulate a limited feedback hybrid beamforming for outdoor environments, including the analog precoding and combining based on a quantization codebook and the digital precoding based on Lagrangian optimization with the mathematical model in detail compared with other algorithms in the literature. The proposed HBF achieves the increase in the spectral efficiency satisfied and works well in all possible and satisfies the quality of the results, such as the increase in the number of users, number of multi-path, the SNR, and the BS antennas. The proposed HBF offers the bit error rate better than other solutions.

The remainder of the paper is organized as follows: Section 2 surveys related research articles, section 3 describes the system model and analyses problem formulation, and section 4 discusses the simulation results. Finally, the conclusion is reported in section 5.

Notations: This paper considers $\mathrm{A}$ and $\mathbf{a}$ as a matrix and a vector. On the other hand, $\mathrm{A}^{\mathrm{H}}, \mathrm{A}^{-1}$, $\mathrm{A}^{\mathrm{T}},|\mathrm{A}|$, and $\|\mathrm{A}\|_{\mathrm{F}}$ represent the Hermitian, inverse, transpose, determinant, and Frobenius norm of a matrix, respectively. $\mathbb{C}$ is the field of complex numbers. Finally, I and [.] denote the identity matrix and the expectation operator.

\section{System model}

On the DL transmission, the micro-cell (MC) is given in Fig. 1, in which multi-users Massive MIMO systems incorporate with the mm-Wave channel based on Proposed Hybrid Beamforming algorithm I (Proposed HBF I). The microcell BS mounts with many antennas $\left(N_{B S}\right)$ that links to RF chains $\left(N_{R F}\right)$. The microcell BS uses a fully connected architecture in which the dedicated RF serves all antennas. 


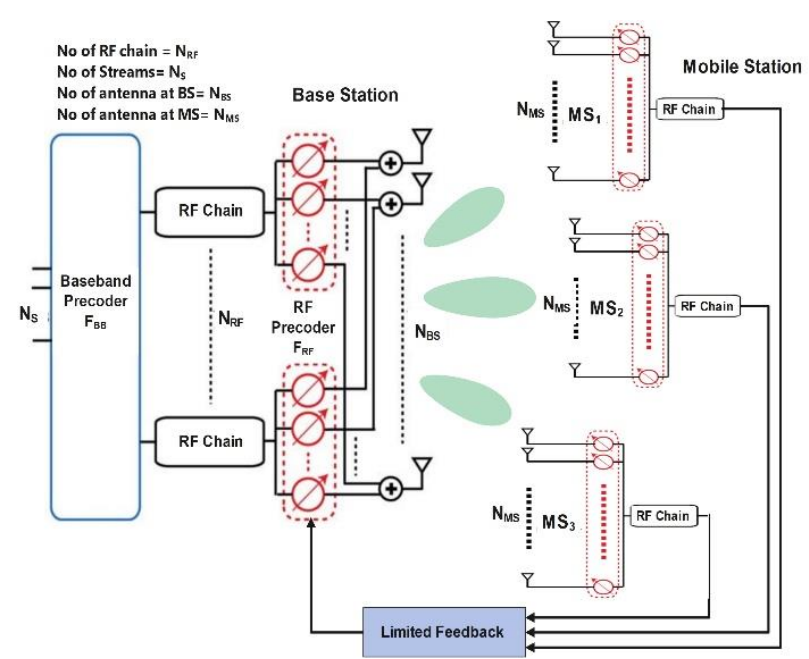

Figure. 1 Proposed hybrid beamforming model

The data streams $\left(N_{S}\right)$ map on all antennas to serve $K$ MSs simultaneously. On the other hand, the mobile station (MS) equips with many antennas $\left(N_{M S}\right)$ and a single RF chain to receive the $N_{S}$ at the receive side. To simplify the problem, we assume that $\left(K=N_{R F}\right)$. It focuses on the FDD implementation, in which the $\mathrm{BS}$ relies on the feedback from users to obtain the precoding details. Here, the microcell BS is operated at $28 \mathrm{GHz}$.

The BS consists of two stages: a low dimensional digital (baseband) precoding $\left(F_{B B} \in \mathbb{C}^{N R F N s}\right)$ and high dimensional analog beamforming $\left(F_{R F} \in \mathbb{C}^{N B S N R F}\right)$. The baseband precoding is applied to the transmitted symbols vector ( $s \in \mathbb{C}^{N s}$ ) as a first stage, and then the analog precoding processes signals as a second stage. Hence, the sampled transmitted signal can be expressed as:

$$
x=F_{R F} F_{B B} S
$$

On the MS side, the received signal between the $\mathrm{BS}$ and $\mathrm{k}^{\text {th }} \mathrm{MSs}$ can be written as:

$$
y_{k}=H_{k} F_{R F} F_{B B} S+n_{k}
$$

In Eq. (2), the received signal can be restated to distinguish the desired signal and the interference as:

$$
\begin{aligned}
& y_{k}=\underbrace{H_{k} F_{R F} F_{B B k} S_{k}}_{\text {Desired signal }}+ \\
& \underbrace{\sum_{j \neq k}^{K} H_{k} F_{R F} F_{B B j} S_{j}}_{\text {Interference signal }}+\underbrace{n_{k}}_{\text {Noise }}
\end{aligned}
$$

Where $n_{k} \in \mathbb{C}^{K}$ is the Gaussian noise vector and $\left(H_{k} \in \mathbb{C}^{N_{M S} N_{B S}}\right)$ is the channel between the BS and the $\mathrm{k}^{\text {th }}$ MSs can be written as:

$$
\begin{aligned}
H= & \sqrt{\frac{N_{B S} N_{M S}}{N_{c l} N_{r a y}}} \sum_{c=1}^{N_{c l}} \sum_{r=1}^{N_{r a y}} u_{c r} u_{M S}\left(\emptyset_{c r}^{M S}, \theta_{c r}^{M S}\right) \\
& u_{B S}\left(\emptyset_{c r}^{B S}, \theta_{c r}^{B S}\right)
\end{aligned}
$$

In (4), NBS and NMS is the number of antennas at BS, and each MS, uMS ( $\phi \mathrm{MS}, \ominus \mathrm{MS})$ and $\mathrm{uBS}$ $(\phi \mathrm{BS}, \Theta \mathrm{BS})$ indicate to array response vectors (steering vectors) for transmitting and receiving side whereas $(\phi \mathrm{BS}, \Theta \mathrm{BS})$ and $\left(\phi \mathrm{MS}, \ominus_{\mathrm{MS}}\right), \quad$ represent azimuth and elevation angles of departure and arrival (AOA, AOD), respectively, and ucr is the complex gain of Nray rays and Ncl clusters for kth MSs. The array response vectors for uniform planar array (UPA) is given by:

$$
\begin{aligned}
& u_{B S}\left(\emptyset_{c r}^{B S}, \theta_{c r}^{B S}\right)= \\
& \frac{1}{\sqrt{N_{B S}}}\left[1, \ldots, e^{-i \frac{2 \pi}{\lambda} d\left(\left(1-N_{B S-w}\right) \cos \left(\theta_{c r}^{B S}\right)\right)}\right. \\
& e^{\left.-i \frac{2 \pi}{\lambda} d\left(\left(1-N_{B S-h}\right) \sin \left(\emptyset_{c r}^{B S}\right) \sin \left(\theta_{c r}^{B S}\right)\right)\right]} \\
& u_{M S}\left(\emptyset_{c r}^{M S}, \theta_{c r}^{M S}\right)= \\
& \frac{1}{\sqrt{N_{M S}}}\left[1, \ldots, e^{-i \frac{2 \pi}{\lambda} d\left(\left(1-N_{M S-w}\right) \cos \left(\theta_{c r}^{M S}\right)\right)}\right. \\
& \mathrm{e}^{\left.-\mathrm{i} \frac{2 \pi}{\lambda} \mathrm{d}\left(\left(1-\mathrm{N}_{\mathrm{MS}-\mathrm{h}}\right) \sin \left(\varnothing_{c r}^{\mathrm{MS}}\right) \sin \left(\theta_{c r}^{\mathrm{MS}}\right)\right)\right]}
\end{aligned}
$$

Where $N_{B S-h}, N_{B S-w}, N_{M S-h}$, and $N_{M S \text { - } w}$ are numbers of uniform planer array (UPA) antennas indexes (height and width) for transmitter and receiver, $\lambda$ is the wavelength and the antenna spacing, which is usually scaled by wavelength $(\mathrm{d}=\lambda / 2)$.

After that, an analog combining $\left(W_{k} \in \mathbb{C}^{N_{M S} K}\right)$ is applied to the received signal. Thus, the estimated signal can be expressed as:

$\hat{x}_{k}=W_{k}^{H} H_{k} F_{R F} F_{B B} S+W_{k}^{H} n_{k}$

In Eq. (7), the received signal can be rewritten to identify the desired signal and the interference as:

$$
\begin{aligned}
\hat{x}_{k} & =\underbrace{W_{k}^{H} H_{k} F_{R F} F_{B B k} S_{k}}_{\text {Desired signal }}+ \\
\underbrace{\sum_{j \neq k}^{K} W_{k}^{H} H_{k} F_{R F} F_{B B j} S_{j}}_{\text {Interference signal }} & +\underbrace{W_{k}^{H} n_{k}}_{\text {Noise }}
\end{aligned}
$$

The sum achievable rate of the system related to $\mathrm{k}^{\text {th }} \mathrm{MSs}$ can be calculated based on the Shannon Theorem, as written:

$$
\mathrm{R}_{\mathrm{k}}=\sum_{\mathrm{k}=1}^{\mathrm{K}} \log _{2}\left[1+\frac{\left|W_{k}^{H} H_{k} F_{R F} F_{B B k} s_{k}\right|^{2}}{\left|\sum_{j \neq k}^{K} W_{k}^{H} H_{k} F_{R F} F_{B B j} s_{j}\right|^{2}+\sigma_{k}^{2}}\right]
$$


Where $\sigma^{2}{ }_{k}$ and $\left|s_{k}\right|^{2}$ are the average noise power and the average total signal power.

\subsection{Problem Formulation of Hybrid Beamforming}

The goal is to minimize the error between the training signal and the estimated signal in Eq. (7). Therefore, we propose the hybrid precoding to achieve it. The optimization problem can be written as:

$$
\underbrace{\operatorname{minimize}}_{F_{R F}, F_{B B}, W} \mathbb{E}\left[\|s-\hat{x}\|_{2}^{2}\right]
$$

Subject to

$$
\begin{gathered}
F_{R F} \in\left\{f_{1}, f_{2 \ldots} f_{Z}\right\} \\
W_{k} \in\left\{W_{1}, W_{2 \ldots} W_{Z}\right\} \\
\|x\|_{2}^{2} \leq P_{t}
\end{gathered}
$$

The constraints in Eqs. (11) and (12) refer to look for the analog precoding and combining in codebooks. At the same time, Eq. (13) indicates the total transmitted power constraint.

Here, the error in Eq. (10) can be converted according to the analog precoding/combining and digital precoding as shown (see Appendix 1):

$$
\begin{aligned}
\mathbb{E}\left[\|s-\hat{x}\|_{2}^{2}\right]= & \mathbb{E}\left[\| s-\left(W_{k}^{H} H_{k} F_{R F} F_{B B} s+\right.\right. \\
& \left.\left.W_{k}^{H} n\right) \|_{2}^{2}\right] \\
= & \mathbb{E}\left[\operatorname { t r } \left(\left(s-W_{k}^{H} H_{k} F_{R F} F_{B B} s+\right.\right.\right. \\
& \left.\left.W_{k}^{H} n\right)\right)\left(s-W_{k}^{H} H_{k} F_{R F} F_{B B} s+\right. \\
& \left.\left.\left.W_{k}^{H} n\right)\right)\right] \\
= & \mathbb{E}\left[\operatorname { t r } \left(s s^{H}-s s^{H} W_{k} H_{k}^{H} F_{R F}^{H} F_{B B}^{H}-\right.\right. \\
& s n^{H} W_{k}+ \\
& W_{k}^{H} H_{k} F_{R F} F_{B B} s s^{H} H_{k}^{H} F_{R F}^{H} F_{B B}^{H} W_{k}- \\
& W_{k}^{H} H_{k} F_{R F} F_{B B} S s^{H}+ \\
& W_{k}^{H} H_{k} F_{R F} F_{B B} s n^{H} W_{k}-W_{k}^{H} n s^{H}+ \\
& W_{k}^{H} n s^{H} H_{k}^{H} F_{R F}^{H} F_{B B}^{H} W_{k}+ \\
& \left.\left.W_{k}^{H} n n^{H} W_{k}\right)\right] \\
= & \operatorname{tr}\left(\left(\mathbb{E}\left[s s^{H}\right]\right)-\right. \\
& 2 R e\left(\mathbb{E}\left[s s^{H}\right] W_{k}^{H} H_{k} F_{R F} F_{B B}\right)+ \\
& W_{k}^{H} H_{k} F_{R F} F_{B B} \mathbb{E}\left[s S^{H}\right] H_{k}^{H} F_{R F}^{H} F_{B B}^{H} W_{k}+ \\
& \left.\sigma^{2} I\right)
\end{aligned}
$$

$$
\begin{aligned}
= & \operatorname{tr}\left(I_{N S}-2 \operatorname{Re}\left(I_{N S} W_{k}^{H} H_{k} F_{R F} F_{B B}\right)+\right. \\
& W_{k}^{H} H_{k} F_{R F} F_{B B} I_{N S} H_{k}^{H} F_{R F}{ }^{H} F_{R F}^{H} W_{k}+ \\
& \sigma^{2} I \\
= & \left\|I_{N S}-W_{k}^{H} H_{k} F_{R F} F_{B B}\right\|_{F}^{2}+ \\
& \text { constant }
\end{aligned}
$$

Also, Eq. (13) can be converted to obtain a complete formulation as:

$$
\begin{aligned}
\|x\|_{2}^{2} \leq P_{t} & =\operatorname{tr}\left(x x^{H}\right) \\
& =\operatorname{tr}\left(F_{R F} F_{B B} S s^{H} F_{B B}^{H} F_{R F}^{H}\right) \\
& =\operatorname{tr}\left(F_{R F} F_{B B} F_{B B}^{H} F_{R F}^{H}\right) \\
& =\left\|F_{R F} F_{B B}\right\|_{2}^{2} \leq P_{t}
\end{aligned}
$$

Hence, Eq. (10) can be reformulated as:

$$
\underbrace{\operatorname{minimize}}_{F_{R F}, F_{B B}, W_{k}}\left\|I_{N s}-W_{k}^{H} H_{k} F_{R F} F_{B B}\right\|_{F}^{2}
$$

Subject to

$$
\begin{gathered}
F_{R F} \in\left\{f_{1}, f_{2 \ldots}, f_{Z}\right\} \\
W_{k} \in\left\{W_{1}, W_{2 \ldots} W_{Z}\right\} \\
\left\|F_{R F} F_{B B}\right\|_{2}^{2} \leq P_{t}
\end{gathered}
$$

It is clear that Eq. (14) replaces data estimation and transmission. However, it depends on $F_{B B}$ and the effective channel (array gain) that is a concatenation of the channel and the analog precoding/combining at the two sides. It is worth noting that each effective channel vector with the $(K x I)$ dimension is smaller than the original channel. It can be written as:

$$
H_{\text {eff }}=W_{k}^{H} H_{k} F_{R F}
$$

The problem in Eq. (14) is a non-convex. Therefore, it is not easy to solve without decomposing into two sub-problems. The nonconvex problem requires designing the digital precoding with the analog beamforming/combining jointly for the optimal system performance. But, the training and feedback overhead are considered, making the optimization problem unpractical and impossible to solve a global optimal. Therefore, we present a two-stage multi-user hybrid precoding.

The central idea of this algorithm is to decompose the beamforming into two stages, each with separate 
constraints and domains to be a simple and lowcomplexity algorithm. Hence, it can be explained as follows.

In the first stage, the goal is a joint design of the analog precoding $F_{R F}$ and the analog combining $W_{k}$ to maximize the desired signal power. However, it neglects the interference between the different users.

In practical systems, it is considered that the channel knowledge may not be perfectly available. Thus, in the simulation of this system, the authors will consider a quantization of the RF precoding. The detailed quantization process is explained as follows:

$$
\theta^{B S}=\left[0, \frac{\pi}{2^{B} R F}, \frac{2 \pi}{2^{B} R F}, \frac{3 \pi}{2^{B} R F}, \ldots \ldots, \frac{\left(2^{B_{R F}}-1\right) \pi}{2^{B} R F}\right]
$$

The maximum array gain (effective channel) is the basis of selecting the pair of the precoding and combining vectors, meaning that the precoding and combining design depends on the effective channel instead of the estimated channel. Finally, this stage does not take into consideration eliminating the interference effect between the users. In the second stage, the effective channel includes information about the design of the receive combiner criteria. To achieve limited feedback for the multi-user MIMO systems, each user feeds back the effective channel and the analog precoding /combining index in the codebook to the base station. Finally, the BS exploits the effective channel to design baseband precoding to cancel the interference. Eq. (14) can be rewritten as:

$$
\underbrace{\text { minimize }}_{F_{R F}, F_{B B}, W_{k}}\left\|I_{N S}-W_{k}^{H} H_{k} F_{R F} F_{B B}\right\|_{F}^{2}
$$

Subject to

$$
\left\|F_{R F} F_{B B}\right\|_{2}^{2} \leq P_{t}
$$

The above equation is a convex problem. However, the total power of the transmitted signal is only considered as a constraint. Therefore, a Lagrangian function is used to attain the best solution. Eq. (14) can be rewritten as:

$$
\begin{array}{r}
\left\|I_{N S}-W_{k}^{H} H_{k} F_{R F} F_{B B}\right\|_{F}^{2} \\
=\operatorname{tr}\left(\left(I_{N S}-W_{k}^{H} H_{k} F_{R F} F_{B B}\right)\right. \\
\left.\quad\left(I_{N S}-W_{k}^{H} H_{k} F_{R F} F_{B B}\right)^{H}\right)
\end{array}
$$

Here, the Lagrangian function is applied, as shown

$$
\begin{aligned}
\mathcal{L}\left(F_{B B}, \beta\right)= & \operatorname{tr}\left(( \mathrm { I } _ { N S } - W ^ { H } H F _ { R F } F _ { B B } ) \left(\mathrm{I}_{N S}-\right.\right. \\
& \left.\left.W^{H} H F_{R F} F_{B B}\right)^{H}\right)+ \\
& \beta\left(\operatorname{tr}\left(F_{R F} F_{B B} F_{R F}^{H} F_{B B}^{H}\right)-P_{t}\right)
\end{aligned}
$$

After that, Eq. (23) has deviated to $\mathrm{F}_{\mathrm{BB}}$, and then it sets 0 to attain the closed-form solution.

$$
\begin{gathered}
F_{B B}=\left(W^{H} H F_{R F} F_{R F}{ }^{H} H^{H} W+\right. \\
\left.\frac{\sigma^{2}}{P_{t}} F_{R F} F_{R F}^{H}\right)^{-1} H^{H} F_{R F}^{H} W \\
F_{B B}=\left(\text { HeffHeff }{ }^{H}+\frac{\sigma^{2}}{P_{t}} F_{R F} F_{R F}^{H}\right)^{-1} H_{e f f}^{H}
\end{gathered}
$$

Fig. 2 summarizes the flowchart followed in the proposed hybrid beamforming.

\section{Simulation results and discussions}

The simulation and analysis take multiple aspects: the BS antennas, the MS antennas, signal-tonoise ratio (SNR), number of users, multi-path channels, and the quantization impact. Our work compares with solutions in [24-26], in addition to the fully digital beamforming, the analog beamforming, and the single-user without interference based on the average achievable rates (bits/s/Hertz) and bit error rate (BER) for all user. The results are more than 1000 random channel implementations on average.

In Fig. 3, the values are plotted as a function of the SNR and average achievable rates in multi-path scenarios. The simulation parameters are five multipath channels, four users, $256 \mathrm{BS}$ antennas, and four MS antennas. The results show that the proposed

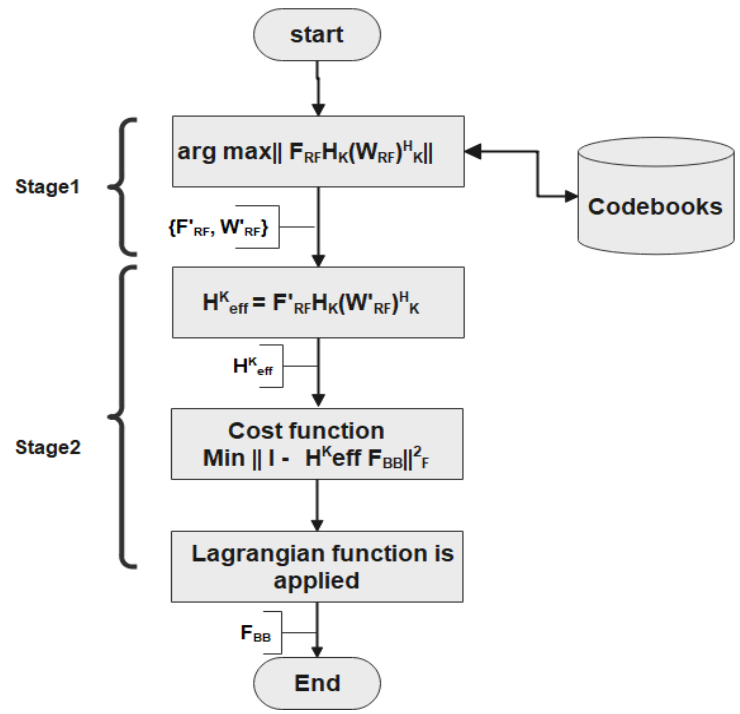

Figure. 2 Flowchart of the proposed HBF I 


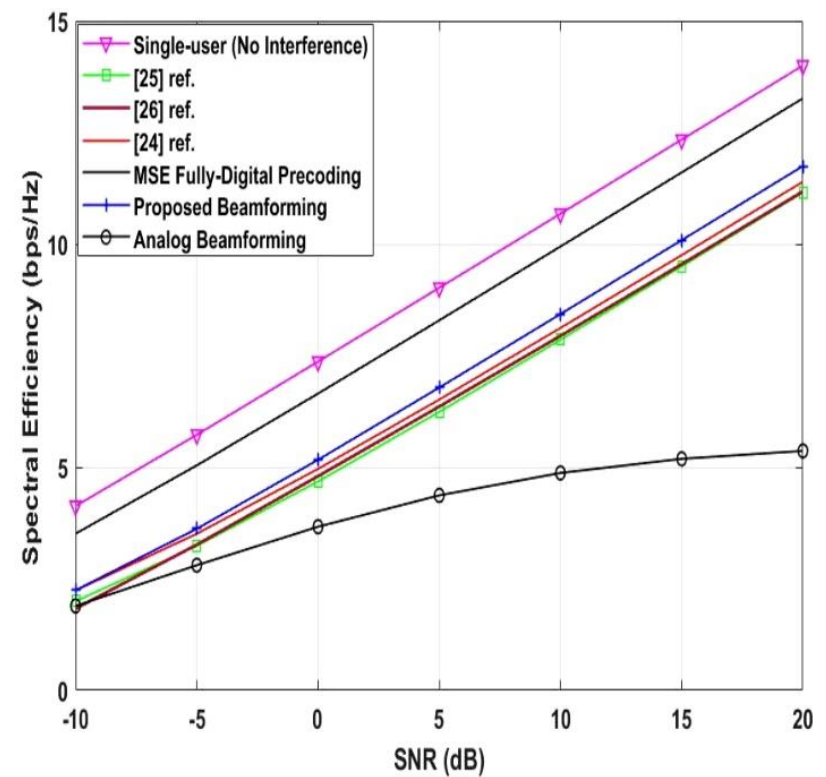

Figure. 3 Spectral efficiency against SNR

HBF I outperforms [24-26]. Also, it achieves spectral efficiency satisfied, such as at $(20 \mathrm{~dB}) \mathrm{SNR}$, it is approximately $(11.75 \mathrm{bps} / \mathrm{Hz})$ while the [24] is $(11.18 \mathrm{bps} / \mathrm{Hz})$, the [25] is $(11.4 \mathrm{bps} / \mathrm{Hz})$, and the [26] is $(11.8 \mathrm{bps} / \mathrm{Hz})$. However, the analog beamforming, the single-user scenario, and the fully-digital beamforming are $(5.37 \mathrm{bps} / \mathrm{Hz}),(14 \mathrm{bps} / \mathrm{Hz})$, and (13.27 bps/Hz).

Fig. 4 illustrates the spectral efficiency against the number of users in the multi-path scenario. It considers the same configuration used in the above figure but sets SNR $10 \mathrm{~dB}$ and MS antennas 16. As observed from the figure, the number of users

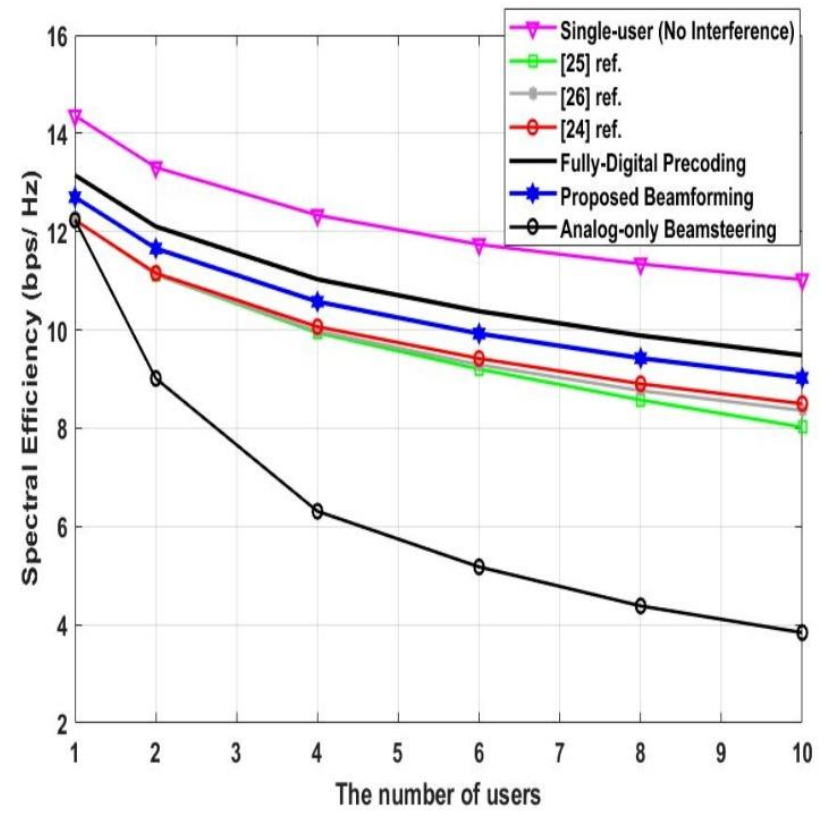

Figure. 4 Spectral efficiency against the number of users

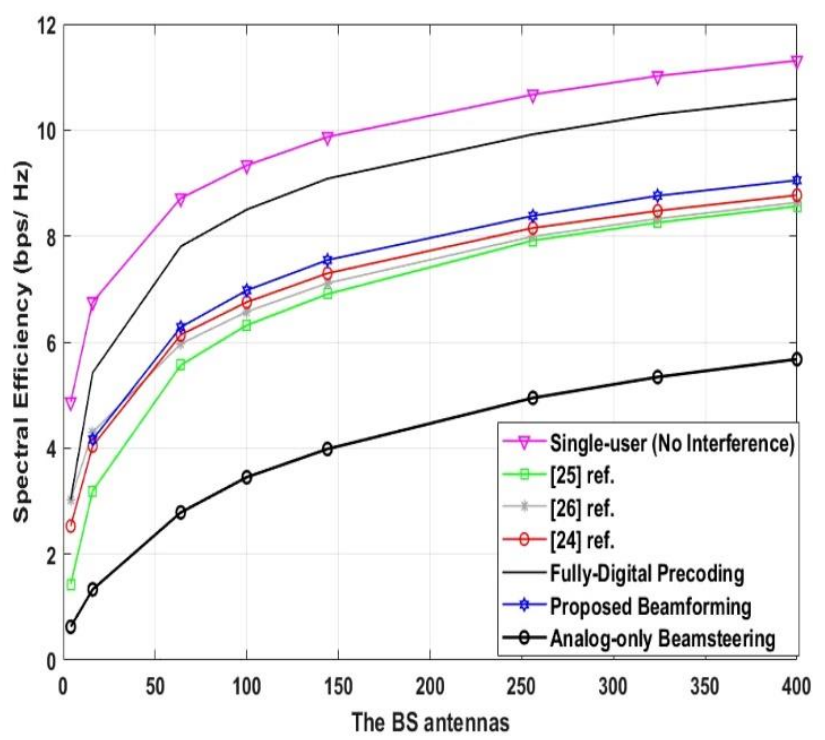

Figure. 5 Spectral efficiency against the BS antennas

increases resulted in a decrease in the spectral efficiency.

In Fig. 5, the spectral efficiency is compared with the BS antennas. It considers the same configuration used in the above figure but sets users four and MS antennas four. The increase in the number of antennas plays a crucial role in obtaining higher spectral efficiency.

Fig. 6 compares the spectral efficiency with multi-path channels, in which 64 BS antennas, ten SNR, four users, and four MS antennas are simulation parameters. In the multi-path scenario, the proposed HBF I illustrates the best performance than other solutions.

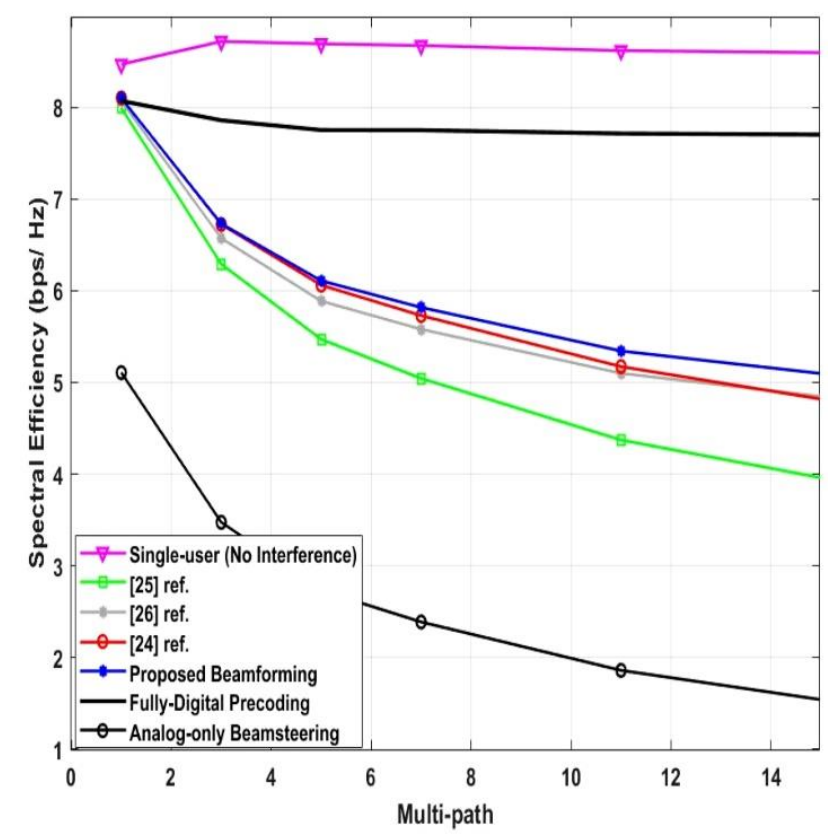

Figure. 6 Spectral efficiency against the number of multipath 


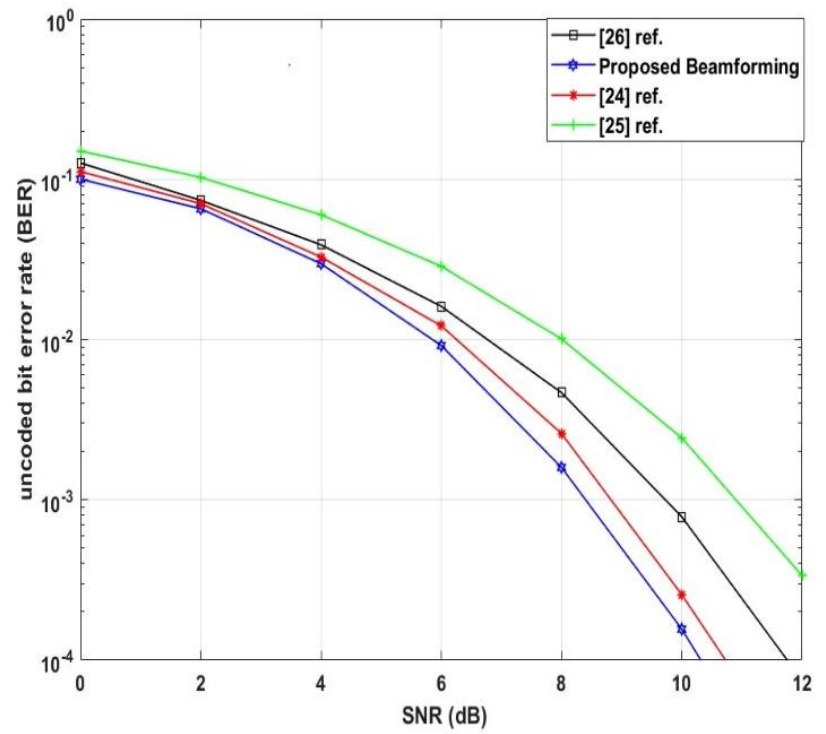

Figure. 7 BER evaluation for the proposed HBF I and competitors

Fig. 7 illustrates the BER performance based on a QPSK modulation, in which four users, 64 BS antennas, and four MS antennas are parameters. It compares the proposed HBF I with other solutions, and the results show that the proposed HBF I is the better one.

It is noted that there are serval points from these results. First, the proposed HBF I provides a significant performance compared to other solutions. The quality of the results is satisfactory for all of the cases. Overall we see the method proposed here easily outperforms the other methods and achieves the increase in the spectral efficiency, as shown in Fig. 3 , such as at $20 \mathrm{~dB}$ SNR, it is approximately 11.75 bps/Hz while the [24] is $11.18 \mathrm{bps} / \mathrm{Hz}$, the [26] is 11.4 $\mathrm{bps} / \mathrm{Hz}$, and the [25] is $11.8 \mathrm{bps} / \mathrm{Hz}$. However, the analog beamforming, the single-user scenario, and the fully-digital beamforming are $5.37 \mathrm{bps} / \mathrm{Hz}, 14$ $\mathrm{bps} / \mathrm{Hz}$, and $13.27 \mathrm{bps} / \mathrm{Hz}$. Therefore, the result confirms that the proposed HBF I can achieve satisfied array gain that leads to the successes of this work compared with other methods, especially at large SNR and the digital stage of the proposed method is effective. The fully digital beamforming achieves the best value of $13.27 \mathrm{bps} / \mathrm{Hz}$ since it requires the dedicated RF equips with the single antenna that leads to more cost and consumption of energy. It is because that the DBF is unpractical. On the contrary, the analog beamforming method achieves the smallest value, $5.37 \mathrm{bps} / \mathrm{Hz}$, because it is the energy-efficient solution that uses inexpensive phase shifters facilitating beam steering. However, it is limited to one data stream, leading to severe performance limitations in the multi-user scenario. Thus, it is not easy to cancel interfering signals.
As observed from Fig. 4, when the number of users increases, the spectral efficiency decreases in the Orthogonal Multiple Access (OMA). However, the decrease rate of the proposed HBF I is acceptable values compared with the other methods. It is due to that the digital stage of the proposed method can effectively adjust the precoding baseband matrix to eliminate the interference between users.

The increase in the number of antennas leads to higher spectral efficiency. However, the spectral efficiency of the proposed HBF I and the fully digital precoding have a smaller gap than other solutions and approximately stays a constant with various BS antennas, meaning that our solution can exploit the number of antennas correctly to increase antenna gains, as shown in Fig. 5.

The proposed solution illustrates the best performance in the multi-path scenario than other solutions, but the ZF beamforming shows the worst performance because it fails in the multi-path environment. It is noted that all hybrid algorithms show similar performance when a single-path scenario. At the same time, there is an increase in multi-path; the proposed solution is better in facing the multi-path since the digital stage of the proposed solution can eliminate interferences between signals while it is worth noting that other solutions fall with increasing multi-path and the gap between curves, as shown in Fig. 6.

In Fig. 7, our method outperforms the alternatives in terms of BER performance. The ZF algorithm achieves a poor performance because it fails in the multi-user environment. As indicated previously, linear detectors achieve near-optimal, but our solution also achieves good BER performance.

Finally, the proposed technique is better than existing techniques because the digital stage of the proposed method can effectively adjust the precoding baseband matrix to eliminate the interference between users and multi-path. Also, our solution can exploit the number of antennas correctly to increase antenna gains based on the quantized codebook. The proposed technique also achieves better BER performance than other solutions because it depends on the proposed error formulation to improve its performance.

\section{Conclusions}

This work studies and discusses the existing algorithms in the literature related to our algorithm with a significant analysis based on performance evaluations. After that, it develops a new hybrid beamforming algorithm for outdoor environments, working in the $5 \mathrm{G}$ networks to achieve the desired 
goals. It achieves an increase in the spectral efficiency satisfied, such as at 20dB SNR, it is approximately $11.75 \mathrm{bps} / \mathrm{Hz}$ while the other solutions are $11.18,11.4$, and $11.8 \mathrm{bps} / \mathrm{Hz}$. Also, it presents work well in all possible, such as increasing the number of users, the number of multi-path, the SNR, and the BS antennas. Also, it offers the BER approximately $10^{-4}$ at $10.3 \mathrm{SNR}$, better than other solutions.

\section{Conflicts of Interest}

The authors declare that there is no conflict of interest regarding the publication of this paper.

\section{Author Contributions}

Conceptualization, M.K.H and N.N.K; methodology, M.K.H; software, M.K.H; validation, M.K.H and N.N.K; formal analysis, M.K.H; investigation, M.K.H and N.N.K; resources, M.K.H; data curation, M.K.H; writing - original draft preparation, M.K.H; writing-review and editing, M.K.H and N.N.K; supervision, N.N.K.

\section{References}

[1] M. Anouar, and S. Larbi, "Array Antenna for Wireless Communication 5G", In: Innovations in Smart Cities Applications, Karabuk, Turkey, pp.931-939,2019.

[2] O. Salih, S. Zeebaree, A. Abdulraheem, R. Zebari, M.Sadeeq, and O. Ahmed, "Evolution of Mobile Wireless Communication", Int. J. Recent Trends Eng. Res., Vol. 05, No. 11, pp. $1-9,2019$.

[3] A. Kumar, and M. Gupta, "A review on activities of fifth generation mobile communication system", Alexandria Engineering Journal, Vol. 57, No. 2, pp. 1125-1135, 2018.

[4] M. Abbasi, and Q. Abbasi, "Development Challenges of Millimeter-Wave 5G Beamformers", Wiley 5G Ref, pp. 1-25, 2020.

[5] M. Taheribakhsh, A. Jafari, M. Peiro, and N. Kazemifard, "5G Implementation: Major Issues and Challenges", In: 2020 25th Int. Comput. Conf. Comput. Soc., Iran, pp. 1-5, 2020.

[6] S. Manap, K. Dimyati, M. Hindia, M. Abu Talip, and R. Tafazolli, "Survey of Radio Resource Management in 5G Heterogeneous Networks", IEEE Access, Vol. 8, pp. 131202-131223, 2020.

[7] N. Al-Falahy, and O. Alani, "Design considerations of ultra dense $5 \mathrm{G}$ network in millimetre wave band", In: Int. Conf.
Ubiquitous Futur. Networks, Milan, Italy, pp. 141-146, 2017.

[8] N. Varshney, and S. De, "Optimum Downlink Beamwidth Estimation in mmWave Communications", IEEE Trans. Commun., Vol. 69, No. 1, pp. 544-557, 2021.

[9] F. Erden, O. Ozdemir, and I. Guvenc, "28 $\mathrm{GHz}$ mmWave Channel Measurements and Modeling in a Library Environment", In: IEEE Radio Wirel. Symp. RWS, San Antonio, TX, USA, pp. 52-55, 2020.

[10] T. Li, and F. Zhao, "A Spectral Efficient Hybrid Precoding Algorithm for MmWave MIMO Systems", Procedia Comput. Sci., Vol. 174, No. 2019, pp. 584-590, 2020.

[11] G. Baghel, M. Swati, and S. Ghosh, MIMO Antennas: A 5G Communication Perspective, Springer, Singapore, pp.1-19, 2021.

[12] M. Tatineni, "Hybrid Precoding/Combining for single-user and Multi-Usersin mm-Wave MIMO systems", Int. J. Innov. Technol. Explor. Eng., Vol. 9, No. 2S3, pp. 134-139, 2019.

[13] R. Chataut, and R. Akl, "Massive MIMO systems for $5 \mathrm{G}$ and beyond networks overview, recent trends, challenges, and future research direction", Sensors (Switzerland), Vol. 20, No. 10, pp. 1-35, 2020.

[14] R. Theodore, H. Robert, D. Robert, and M. James, MillimeterWave Wireless Communications, Pearson Education, United States, 2015.

[15] D. Nguyen, L. Le, T. Ngoc, and R. Heath, "Hybrid MMSE Precoding and Combining Designs for mmWave Multiuser Systems", IEEE Access, Vol. 5, pp. 19167-19181, 2017.

[16] M. AbouYassin, and H. Abdallah, "Hybrid Beamforming in Multiple User Massive Multiple Input Multiple Output 5G Communications System", In: 2020 7th Int. Conf. Electr. Electron. Eng. ICEEE 2020, Antalya, Turkey, pp. 215-220, 2020.

[17] Z. Zhou, N. Ge, Z. Wang, and S. Chen, "Hardware-efficient hybrid precoding for millimeter wave systems with multi-feed reflectarrays", IEEE Access, Vol. 6, No. c, pp. 6795-6806, 2018.

[18] N. Souto, J. Silva, J. Pavia, and M. Ribeiro, "An alternating direction algorithm for hybrid precoding and combining in millimeter wave MIMO systems", Phys. Commun., Vol. 34, pp. 165-173, 2019.

[19] O. Alluhaibi, Q. Ahmed, C. Pan, and H. Zhu, "Hybrid Digital-to-Analog Beamforming Approaches to Maximise the Capacity of mm- 
Wave Systems", In: IEEE Veh. Technol. Conf., Sydney, NSW, Australia, pp. 0-4, 2017.

[20] K. Duan, H. Du, and Z. Wu, "Hybrid Alternating Precoding and Combining Design for mmWave Multi-User MIMO Systems", In: 2018 IEEE/CIC Int. Conf. Commun. ICCC 2018, Beijing, China, pp. 217-221, 2019.

[21] B. Seo, "Hybrid combiner design for downlink massive MIMO systems", ETRI J., pp.333-400, 2020.

[22] A. Uwaechia, and N. Mahyuddin, "A comprehensive survey on millimeter wave communications for fifth-generation wireless networks: Feasibility and challenges", IEEE Access, Vol. 8, pp. 62367-62414, 2020.

[23] C. Najaraju, G. Chandana, B. Kumar, and C. Kumar, "Development of Hybrid Pre-coding Technique for Mimo Systems Based on Kalman Filter", In: 3rd International Conference on Communications and Cyber Physical Engineering, Singapore, pp. 451460, 2021.

[24] M. Shaheenbanu, and M. Suma, "Investigation on hybrid precoding for multi-user MIMO systems", In: 20195 th IEEE Int. WIE Conf. Electr. Comput. Eng. WIECON-ECE 2019 - Proc., Bangalore, India, pp. 1-4, 2019.

[25] M. Shehata, A. Mokh, M. Crussière, M. Hélard, and P. Pajusco, "On the equivalence of hybrid beamforming to full digital zero forcing in mmWave MIMO”, In: 26th Internatinal Conf. Telecommun. (ICT 2019), Hanoi, Vietnam, 2019.

[26] S. Said, W. Saad, and M. Shokair, "MMSE algorithm based two stages hybrid precoding for millimeter wave massive MIMO systems", Analog Integr. Circuits Signal Process., Vol. 98, No. 3, pp. 565-573, 2019.

[27] T. Lin, J. Cong, Y. Zhu, J. Zhang, and K. Ben Letaief, "Hybrid Beamforming for Millimeter Wave Systems Using the MMSE Criterion", IEEE Trans. Commun., Vol. 67, No. 5, pp. 3693-3708, 2019.

[28] X. Zhang, Matrix analysis and applications, Cambridge University Press, United Kingdom, 2017.

Appendix 1: Matrix Properties

According to [28]

1. $\mathbb{E}\left[s s^{H}\right]=I_{N S}$

2. $\mathbb{E}\left[n s^{H}\right]=\mathbb{E}\left[s n^{H}\right]=0$

3. $\|x\|_{2}^{2}=\operatorname{tr}\left(x x^{H}\right)$
4. $x+x^{H}=2 \operatorname{Re}(x)$

5. $\|x-y\|_{F}^{2}=\operatorname{tr}(\mathrm{x}-y)(\mathrm{x}-y)^{H}$ 